\title{
Mulheres climatéricas no ambiente de trabalho: Revisão integrativa da literatura
}

\author{
Climate women in the workplace: Integrative literature review \\ Mujeres climatéricas en el lugar de trabajo: Revisión integradora de la literatura
}

Recebido: 24/06/2021 | Revisado: 01/07/2021 | Aceito: 05/07/2021 | Publicado: 16/07/2021

\author{
Giuliana Fernandes e Silva \\ ORCID: https://orcid.org/0000-0002-1130-2587 \\ Centro Universitário Anhanguera de Niterói, Brasil \\ E-mail: giulianafernandes@hotmail.com \\ Larissa Trigoli Lima \\ ORCID: https://orcid.org/0000-0001-8813-078X \\ Centro Universitário Anhanguera de Niterói, Brasil \\ E-mail: lariissa.trigoli.lima@gmail.com \\ Rafaella Dias de Carvalho \\ ORCID: https://orcid.org/0000-0002-6657-8628 \\ Centro Universitário Anhanguera de Niterói, Brasil \\ E-mail: rafaelladias2010@ hotmail.com \\ Pedro Paulo Corrêa Santana \\ ORCID: https://orcid.org/0000-0002-4470-9746 \\ Centro Universitário Anhanguera de Niterói, Brasil \\ E-mail: psantana.uff@gmail.com \\ Luiz Carlos Moraes França \\ ORCID: https://orcid.org/0000-0002-6370-115X \\ Centro Universitário Anhanguera de Niterói, Brasil \\ E-mail: lcmoraesfranca@ hotmail.com
}

\begin{abstract}
Resumo
O presente estudo teve como objetivo investigar as evidências científicas acerca das influências do climatério no cotidiano de trabalho de mulheres que vivenciaram essa fase. Trata-se de uma revisão integrativa da literatura realizado entre maio e setembro de junho de 2020, com publicações entre o período de 2010 a 2020, nos idiomas português, inglês e espanhol que abordassem a temática nas bases de dados PUBMED, Biblioteca Virtual em Saúde e Literatura Latino Americana. Os resultados dos estudos evidenciam o climatério como uma fase complexa na percepção das mulheres e apontam a dificuldade em conviver com os sintomas no ambiente de trabalho. Sinalizaram também, que neste cenário inferem o aumento sobre a intensidade dos sintomas. Abordam ainda sobre a qualidade de vida das mulheres e questões relativas à vida sexual destacando a forma negativa vivenciada nesta fase. O conhecimento da população feminina acerca do climatério ainda é deficiente e/ou escasso e a sintomatologia desta fase pode afetar a capacidade nas atividades do trabalho de algumas mulheres. Essa condição reflete em fragilidades quanto a sua autopercepção para o desempenho de seus afazeres. E conclui-se que o estudo contribui com evidências atuais possibilitando criação de estratégias aos profissionais na assistência às demandas das mulheres. É necessário maior esclarecimento para que as mulheres tenham uma compreensão sobre a fase fisiológica do climatério.
\end{abstract}

Palavras-chave: Enfermagem; Climatério; Promoção da saúde; Mulheres trabalhadoras.

\begin{abstract}
The present study aimed to investigate the scientific evidence about the influences of climacteric illness on the daily work of women who have experienced this phase. This is an integrative literature review conducted between May and September of June 2020, with publications between 2010 and 2020, in Portuguese, English and Spanish languages that addressed the topic in the databases PUBMED, Virtual Health Library and Latin American Literature. The results of the studies show the climacteric period as a complex phase in the perception of women and point to the difficulty in living with the symptoms in the workplace. They also pointed out that in this scenario they infer an increase in the intensity of the symptoms. They also approach the quality of life of women and issues related to sex life, highlighting the negative way they experience in this phase. The female population's knowledge about the climacteric period is still deficient and/or scarce, and the symptoms of this phase can affect the ability of some women to perform work activities. This condition reflects in weaknesses regarding their self-perception for the performance of their tasks. And it is concluded that the study contributes with current evidence enabling the creation of strategies for professionals in assisting the demands of women. Greater clarification is needed so that women have an understanding of the physiological phase of climacteric.
\end{abstract}

Keywords: Nursing; Climacteric; Health promotion; Women working. 


\begin{abstract}
Resumen
El presente estudio tenía como objetivo investigar la evidencia científica sobre las influencias del climaterio en el trabajo diario de las mujeres que han experimentado esta fase. Se trata de una revisión bibliográfica integradora realizada entre mayo y septiembre de junio de 2020, con publicaciones entre 2010 y 2020, en los idiomas portugués, inglés y español que abordaron el tema en las bases de datos PUBMED, Biblioteca Virtual de Salud y Literatura Latinoamericana. Los resultados de los estudios muestran el periodo climatérico como una fase compleja en la percepción de las mujeres y señalan la dificultad de convivir con los síntomas en el trabajo. También señalaron que en este escenario se infiere un aumento de la intensidad de los síntomas. También abordan la calidad de vida de las mujeres y las cuestiones relacionadas con la vida sexual destacando la forma negativa que se experimenta en esta fase. El conocimiento de la población femenina sobre el periodo climatérico es todavía deficiente y/o escaso y los síntomas de esta fase pueden afectar a la capacidad en las actividades laborales de algunas mujeres. Esta condición se refleja en las debilidades respecto a su autopercepción para el desempeño de sus tareas. Se concluye que el estudio contribuye con evidencias actuales que permiten la creación de estrategias para los profesionales en la asistencia a las demandas de las mujeres. Se necesita una mayor aclaración para que las mujeres comprendan la fase fisiológica del climaterio.

Palabras clave: Enfermería; Climaterio; Promoción de la salud; Mujeres trabajadoras.
\end{abstract}

\title{
1. Introdução
}

O climatério é uma etapa biológica da vida da mulher e compreende uma fase de transição entre o período reprodutivo para não reprodutivo (Schumalfuss, Sehnem, \& Rossetto, 2020). Este estágio constitui um processo natural na vida das mulheres, embora algumas vivenciem sem sintomatologia, outras podem apresentar sintomas que variam de acordo com a intensidade e diversidade. Nesse contexto, segundo a Organização Mundial da Saúde (OMS), o climatério é definido como uma fase biológica e não como um processo patológico da mulher, podendo desencadear vários sintomas como vasomotores, psicológicos, urogenitais, sexuais e outros, advindos do hipoestrogenismo.

Segundo dados do IBGE (2010) a projeção para o ano de 2021 aponta que as mulheres constituem a maioria da população brasileira, correspondente a 51,12\%. Somando a esta realidade, estudos realizados (PNAD contínua, 2018) em 2017, revelam que as mulheres são a maioria expressiva na população idosa do Brasil (56\% dos idosos), nessa perspectiva as mulheres vivem mais do que os homens, imprimindo uma importante característica a população idosa que é a sua feminilização.

Visto isso, com o aumento da expectativa de vida em mulheres e o papel social e profissional que elas vêm conquistando nos últimos anos, tornaram-se necessários o entendimento do período do climatério e o acolhimento adequado dessas mulheres, à medida que essa fase ocorre em plena vida produtiva da mulher.

Considerando esta realidade, a maioria das mulheres irão vivenciar o climatério, entretanto, ainda que seja uma fase biológica, estudos (Silva, Moura, Almeida, Sá e Queiroz, 2015; Silva, Rocha \& Caldeira, 2018) apontam que muitas mulheres vivenciam conflitos nesta fase e a associam com o envelhecimento, destacando repercussões negativas.

Muitas mulheres não conhecem ou não identificam a maior parte das alterações hormonais, fisiológicas e emocionais envolvidas no processo de declínio da produção hormonal e término de ciclos menstruais. Os desconhecimentos dessa fase podem estar associados a outros conflitos socioeconômicos e culturais que, somados ao período da vida e à individualidade dessas mulheres, agravam seu estado físico e emocional. Como as políticas de saúde da mulher seguem focadas na manutenção do ciclo gravídico-puerperal, muitas vezes, as mulheres que estão na fase climatérica não são consideradas no contexto dos serviços de saúde (Schumalfuss et al, 2020).

O climatério e a menopausa geralmente não estão associados ao local de trabalho ou à saúde e segurança dos trabalhadores, em contrapartida, muitos problemas podem surgir devido às condições de trabalho que essa mulher pode enfrentar juntamente com a fase (Bariola, Jack, Pitts, Riach, \& Sarrel, 2017). O estresse no trabalho é um fator cada vez mais importante do crescente número de mulheres atuando no ambiente de trabalho.

Segundo Matsuzaki, Uemura, e Yasui (2014), existem inter-relações entre trabalho e sintomas da menopausa, como cansaço, distúrbios do sono, falta de memória e concentração e humor deprimido. Dessa forma, Hunter, Hardy, Norton, e 
Griffiths (2016) descrevem a percepção de algumas mulheres que identificam ser difícil controlar os sintomas no emprego e que certas situações no ambiente de trabalho, como reuniões formais, trabalho com homens e colegas mais jovens, e exercendo suas atividades em locais quentes ou ambientes mal ventilados, aumentam a intensidade dos sintomas. Esses sintomas podem afetar a capacidade no exercício de algumas mulheres ou a autopercepção da capacidade de trabalho.

Sendo assim, o climatério enquanto fase de vida passa a ser um desafio para as autoridades e para as mulheres que vivenciam, já que a prevenção adequada pode melhorar a qualidade e expectativa de vida das mulheres climatéricas. Observase na literatura, escassez de estudos que abordem o climatério interligado com o ambiente de trabalho. Desse modo, considerando essa problemática, o presente estudo teve como objetivo investigar as evidências científicas acerca das influências do climatério no cotidiano de trabalho de mulheres que vivenciaram essa fase.

\section{Metodologia}

Trata-se de uma revisão integrativa de literatura, desenvolvida através de uma pesquisa bibliográfica. Segundo Ercole, Melo e Arcoforado (2014), a revisão integrativa da literatura insere-se no modelo da Prática Baseada em Evidências, em termos de uma sistematização da metodologia de busca, seleção, avaliação, interpretação e apresentação dos resultados. Tem como objetivo sintetizar resultados obtidos em pesquisas sobre um tema ou questão, de maneira sistemática, ordenada e abrangente. Sendo possível usar informações de diferentes fontes para revisar teorias, identificar lacunas e buscar novas estratégias para o problema abordado.

Após a definição do tema do estudo, realizou-se um levantamento bibliográfico nas bases de dados Literatura LatinoAmericano e do Caribe em Ciências da Saúde (LILACS) e Public/Publish-Mediline (PUBMED) e na Biblioteca Virtual em Saúde (BVS). As buscas foram realizadas nos meses de maio a setembro de 2020. Para o estudo, foi utilizado a combinação dos descritores: Mulheres Trabalhadoras; Climatério; Enfermagem; Promoção em Saúde; conforme apresentação do vocabulário contido nos Descritores em Ciências da Saúde (Decs), validados pela BVS e cruzadas entre si por meio do operador booleano AND.

Como critérios de inclusão foram selecionados artigos nos idiomas português, inglês e espanhol, publicados entre os anos de 2010 e 2020 e que estivessem disponíveis na íntegra. Foram excluídos os artigos repetidos nas bases de dados e que não atendessem à temática. A partir da aplicação da estratégia PICO e dos descritores utilizados foram encontrados 534 artigos, após a aplicação dos critérios de inclusão e exclusão totalizou-se uma amostra parcial 48 artigos, depois de uma leitura mais precisa aderiu-se a um total final de 19 publicações que compõe as amostras desta revisão. Esse refinamento pode ser exemplificado no fluxograma da Figura 1 a seguir. 
Figura 1. Fluxograma para seleção dos artigos para elaboração da revisão integrativa, 2021.

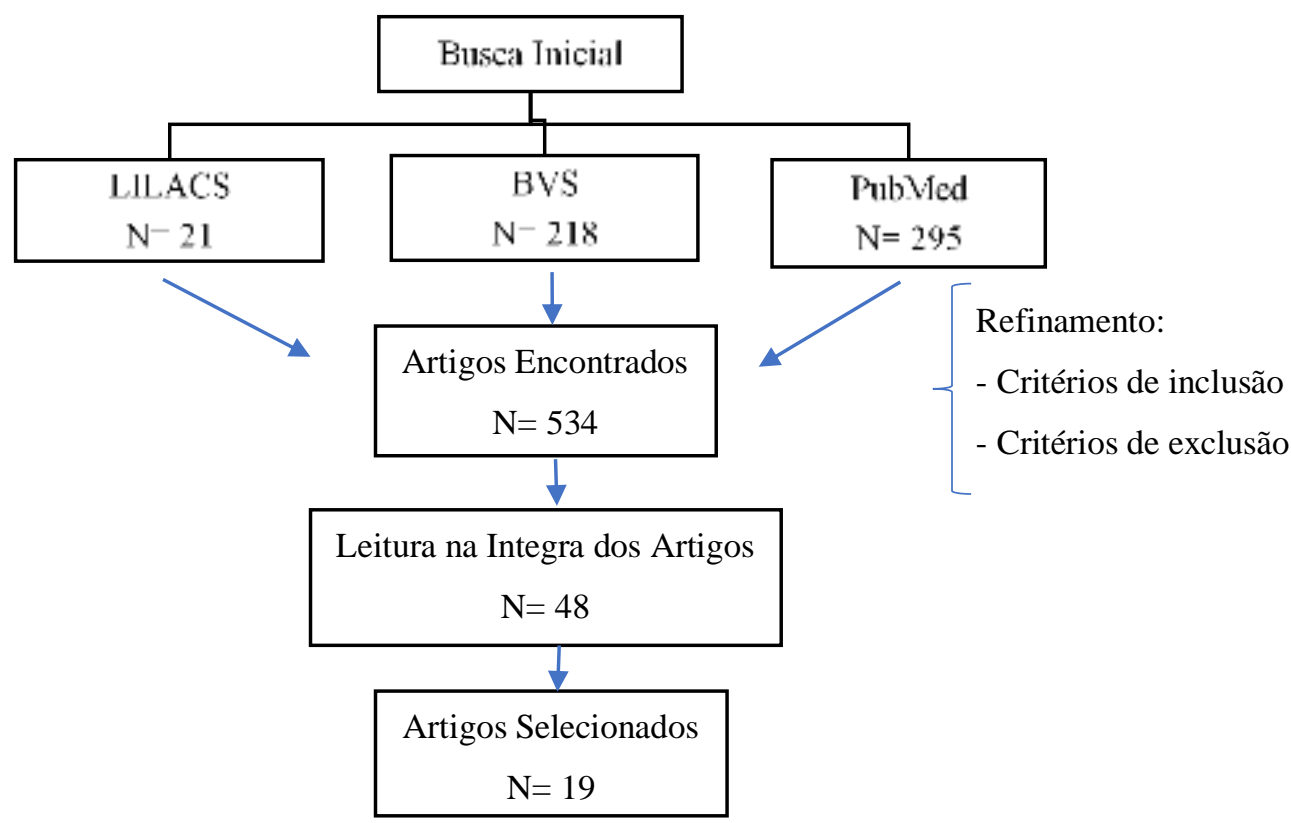

Fonte: Autores (2021).

O fluxograma acima apresenta as etapas do processo de pesquisa e seleção das obras escolhidas para esta revisão, demonstrando a ordem de filtro e resultados apresentados. Considerando os critérios apontados a revisão foi baseada na análise de 19 artigos que foram organizados no Quadro 1 a seguir.

Quadro 1. Artigos selecionados na revisão de literatura do estudo, 2021.

\begin{tabular}{|c|c|c|c|c|c|}
\hline Código & $\begin{array}{l}\text { Bases de } \\
\text { Dados }\end{array}$ & $\begin{array}{l}\text { Ano de } \\
\text { Publicação }\end{array}$ & Autores & Título & Objetivo (s) \\
\hline A1 & LILACS & 2019 & $\begin{array}{l}\text { MAMEDE } \\
\text { SILVA } \\
\text { OLIVEIRA B da } \text { S }\end{array}$ & $\begin{array}{l}\text { Conhecimento e sentimentos } \\
\text { das mulheres climatéricas sobre } \\
\text { a doença coronariana }\end{array}$ & $\begin{array}{l}\text { Descrever o impacto da descoberta da } \\
\text { doença coronariana no cotidiano das } \\
\text { mulheres climatéricas. }\end{array}$ \\
\hline $\mathrm{A} 2$ & LILACS & 2018 & $\begin{array}{l}\text { SILVA VH; ROCHA } \\
\text { JSB; } \quad \text { CALDEIRA } \\
\text { AP; }\end{array}$ & $\begin{array}{l}\text { Fatores associados à } \\
\text { autopercepção negativa de } \\
\text { saúde em mulheres climatéricas }\end{array}$ & $\begin{array}{l}\text { Investigar a prevalência e os fatores } \\
\text { associados à autopercepção negativa de } \\
\text { saúde em mulheres climatéricas } \\
\text { cadastradas na Estratégia Saúde da } \\
\text { Família em um centro urbano } \\
\text { brasileiro. }\end{array}$ \\
\hline A3 & LILACS & 2018 & $\begin{array}{l}\text { de ARRUDA GT; da } \\
\text { CAMPO GS; BRAZ } \\
\text { MM; }\end{array}$ & $\begin{array}{l}\text { Incontinência urinária e } \\
\text { disfunções sexuais em mulheres } \\
\text { climatéricas de um grupo de } \\
\text { promoção à saúde }\end{array}$ & $\begin{array}{l}\text { Analisar a ocorrência e as } \\
\text { características de IU e DSF em } \\
\text { mulheres climatéricas de um grupo de } \\
\text { promoção à saúde de uma cidade do } \\
\text { interior do Rio Grande do Sul. }\end{array}$ \\
\hline A4 & BVS & 2016 & $\begin{array}{l}\text { MARTÍNEZ- } \\
\text { GARDUÑO MD; } \\
\text { OLIVIOS-RUBIO }\end{array}$ & $\begin{array}{l}\text { Intervención educativa de } \\
\text { enfermeira para fomentar el } \\
\text { autocuidado de la mujer durante }\end{array}$ & $\begin{array}{l}\text { Evaluar las intervenciones educativas } \\
\text { de enfermería para el autocuidado em } \\
\text { las mujeres durante el climatério. }\end{array}$ \\
\hline
\end{tabular}




\begin{tabular}{|c|c|c|c|c|c|}
\hline & & & $\begin{array}{l}\text { M; GÓMEZ- } \\
\text { TORRES D; CRUZ- } \\
\text { BELLO P. }\end{array}$ & el climatério & \\
\hline A5 & BVS & 2016 & $\begin{array}{l}\text { FREITAS ER; } \\
\text { BARBOSA AJG; et } \\
\text { al; }\end{array}$ & $\begin{array}{l}\text { Educação em saúde para } \\
\text { mulheres no climatério: } \\
\text { impactos na qualidade de vida }\end{array}$ & $\begin{array}{l}\text { Avaliar os efeitos de um processo } \\
\text { multidisciplinar de educação em saúde } \\
\text { (ES) na qualidade de vida (QV) de } \\
\text { mulheres climatéricas. }\end{array}$ \\
\hline A6 & BVS & 2015 & $\begin{array}{l}\text { ALVES ERP; } \\
\text { da COSTA AM; et al; }\end{array}$ & $\begin{array}{l}\text { Climatério: a intensidade dos } \\
\text { sintomas e o desempenho } \\
\text { sexual }\end{array}$ & $\begin{array}{l}\text { Verificar a associação entre a } \\
\text { intensidade dos sintomas no climatério } \\
\text { e o padrão de desempenho sexual de } \\
\text { mulheres neste período do ciclo vital }\end{array}$ \\
\hline A7 & LILACS & 2014 & $\begin{array}{l}\text { MIRANDA } \\
\text { FERREIRA } \\
\text { CORRENTE JE; }\end{array}$ & $\begin{array}{l}\text { Qualidade de vida em mulheres } \\
\text { no climatério atendidas na } \\
\text { Atenção Primária }\end{array}$ & $\begin{array}{l}\text { Avaliar a qualidade de vida de } \\
\text { mulheres na fase do climatério, com ou } \\
\text { sem uso da terapia de reposição } \\
\text { hormonal (TRH). }\end{array}$ \\
\hline A8 & LILACS & 2011 & $\begin{array}{l}\text { SANTOS JS; } \\
\text { FIALHO AVM; } \\
\text { RODRIGUES DP; }\end{array}$ & $\begin{array}{l}\text { Influências das famílias no } \\
\text { cuidado às } \\
\text { climatéricas }\end{array}$ & $\begin{array}{l}\text { compreender as influências das } \\
\text { famílias no cuidado à mulher } \\
\text { climatérica. }\end{array}$ \\
\hline A9 & BVS & 2010 & $\begin{array}{l}\text { VALENÇA CN; } \\
\text { FILHO JMN; } \\
\text { GERMANO RM; }\end{array}$ & $\begin{array}{l}\text { Mulher no climatério: reflexões } \\
\text { sobre desejo sexual, beleza e } \\
\text { feminilidade }\end{array}$ & $\begin{array}{l}\text { refletir sobre desejo sexual, beleza e } \\
\text { feminilidade da mulher nessa fase. }\end{array}$ \\
\hline A10 & LILACS & 2011 & $\begin{array}{l}\text { MEMBRIVE JM; } \\
\text { GRANERO- } \\
\text { MOLINA J; et al; }\end{array}$ & $\begin{array}{l}\text { Qualidade de vida em mulheres } \\
\text { climatéricas que trabalham no } \\
\text { sistema sanitário e educativo }\end{array}$ & $\begin{array}{l}\text { determinar a associação entre a } \\
\text { atividade profissional e a qualidade de } \\
\text { vida percebida em mulheres } \\
\text { climatéricas que trabalham em Saúde e } \\
\text { Educação. }\end{array}$ \\
\hline A11 & PubMed & 2018 & $\begin{array}{l}\text { MARLATT KL; } \\
\text { REDMAN ABLM; }\end{array}$ & $\begin{array}{l}\text { A qualitative assessment of } \\
\text { health behaviors and } \\
\text { experiences during menopause: } \\
\text { A cross-sectional, observational } \\
\text { study }\end{array}$ & $\begin{array}{l}\text { To assess the health and lifestyle } \\
\text { behaviors of middle-aged women } \\
\text { during the menopause transition, as } \\
\text { well as to inform the development of } \\
\text { interventions designed to combat } \\
\text { menopause symptoms and improve } \\
\text { quality of life during and after } \\
\text { menopause. }\end{array}$ \\
\hline A12 & PubMed & 2018 & $\begin{array}{l}\text { RACZKIEWICZ D; } \\
\text { Alfred OWOC } \\
\text { A; ANNA } \\
\text { WIERZBIŃSKA- } \\
\text { STĘPNIAK A; } \\
\text { BOJAR I; }\end{array}$ & $\begin{array}{l}\text { Metabolic syndrome in peri - } \\
\text { and postmenopausal women } \\
\text { performing intellectual work }\end{array}$ & $\begin{array}{l}\text { To analyze the frequency of MS and its } \\
\text { criteria in perimenopausal and } \\
\text { postmenopausal women doing } \\
\text { intellectual work, as well as selected } \\
\text { factors on which metabolic syndrome } \\
\text { depend. }\end{array}$ \\
\hline A13 & PubMed & 2017 & JAFARI M; SEIFI B; & $\begin{array}{l}\text { Risk Assessment: Factors } \\
\text { Contributing to Discomfort for }\end{array}$ & To review the Factors contributing to \\
\hline
\end{tabular}




\begin{tabular}{|c|c|c|c|c|c|}
\hline & & & HEIDARI M; & $\begin{array}{lll}\text { Menopausal Women in } & \text { in } \\
\text { Workplace } & \end{array}$ & $\begin{array}{l}\text { discomfort for menopausal women in } \\
\text { workplace and the perceived effects of } \\
\text { working conditions on menopausal } \\
\text { symptoms, and to produce } \\
\text { recommendations for managers and } \\
\text { women. }\end{array}$ \\
\hline A14 & PubMed & 2015 & $\begin{array}{l}\text { YAZDKHASTI M; } \\
\text { SIMBAR M; ABDI } \\
\text { F; }\end{array}$ & $\begin{array}{l}\text { Empowerment and coping } \\
\text { strategies in menopause } \\
\text { women: a review }\end{array}$ & $\begin{array}{l}\text { To review the empowerment and } \\
\text { coping strategies in menopause } \\
\text { women. }\end{array}$ \\
\hline A15 & PubMed & 2017 & $\begin{array}{l}\text { GUJSKI M; PINKAS } \\
\text { J; et al; }\end{array}$ & $\begin{array}{l}\text { STRESS AT THE PLACE OF } \\
\text { WORK AND COGNITIVE } \\
\text { FUNCTIONS } \\
\text { WOMEN AMONG } \\
\text { INTELLECTUAL PERFORING } \\
\text { DURING PERI- AND POST- } \\
\text { MENOPAUSAL PERIOD }\end{array}$ & $\begin{array}{l}\text { The analysis of the relationship } \\
\text { between stress at work and results of } \\
\text { cognitive functions amongst women, } \\
\text { at peri- and post-menopausal age, } \\
\text { performing intellectual work. }\end{array}$ \\
\hline A16 & PubMed & 2018 & $\begin{array}{l}\text { ATRIAN } \\
\text { KM; SOLHI M; et al; }\end{array}$ & $\begin{array}{l}\text { Association of self-care status } \\
\text { with some relevant factors in } \\
\text { middle-aged women in their } \\
\text { early menopausal stage }\end{array}$ & $\begin{array}{l}\text { To determine the association of self- } \\
\text { care status with some relevant factors } \\
\text { in Kashanian middle-aged women in } \\
\text { their early menopausal stage. }\end{array}$ \\
\hline A17 & PubMed & 2018 & $\begin{array}{l}\text { AFSAHRI } \quad P \text {; } \\
\text { SALEHNEJAD P; et } \\
\text { al; }\end{array}$ & $\begin{array}{l}\text { Do Sleeping Disorders Impair } \\
\text { Sexual Function in Married } \\
\text { Iranian Women of Reproductive } \\
\text { Age? Results from a Cross- } \\
\text { Sectional Study }\end{array}$ & $\begin{array}{l}\text { The relationship between sleep quality } \\
\text { and sexual function among Iranian } \\
\text { women. }\end{array}$ \\
\hline A18 & PubMed & 2019 & $\begin{array}{l}\text { THOMAS } \\
\text { HN; HAMM M; et al; }\end{array}$ & $\begin{array}{l}\text { Body Image, Attractiveness, } \\
\text { and Sexual Satisfaction Among } \\
\text { Midlife Women: A Qualitative } \\
\text { Study }\end{array}$ & $\begin{array}{l}\text { To explore how body image relates to } \\
\text { sexual function and satisfaction in } \\
\text { midlife women. }\end{array}$ \\
\hline A19 & PubMed & 2012 & $\begin{array}{l}\text { REZAEI N; HAKIMI } \\
\text { S; ALI M; }\end{array}$ & $\begin{array}{l}\text { Predictors of health-related } \\
\text { quality of life in } \\
\text { postmenopausal women: a } \\
\text { population-based study }\end{array}$ & $\begin{array}{l}\text { to examine the associations between } \\
\text { health utility (HU), health-related } \\
\text { quality of life (HRQoL), and patient } \\
\text { characteristics in postmenopausal } \\
\text { osteoporotic (PMO) women. }\end{array}$ \\
\hline
\end{tabular}

Fonte: Autores (2021).

\section{Resultados e Discussão}

Atualmente o crescente envelhecimento da população é um dos maiores desafios que a sociedade enfrenta, juntamente com a industrialização. Apesar do número de mulheres trabalhadoras terem aumentado, as taxas de emprego são geralmente mais baixas entre mulheres e homens mais velhos. Outro problema refere-se a diferença salarial no trabalho remunerado, além 
do destaque de que as mulheres não apresentam a mesma participação no mercado de trabalho que os homens, dado que apenas 43,20\% com mais de 1 ano estavam trabalhando em 2014, ao passo que 56,80\% dos homens estavam ocupados (IBGE, 2015).

As fontes de publicação foram variadas, dentre os quais destacamos periódicos da área de saúde coletiva/saúde pública (A2, A5, A7, A9, A11) que colaboraram com 26,33\%, e os da área da Enfermagem (A1, A4, A6, A8, A10), representados por cinco revistas diferentes que, também contribuíram com a amostra.

Entre as publicações, verifica-se que a maioria $(57,90 \%)$ das produções científicas tem origem Internacional, sendo 26,34\% no Irã, 15,78\% na Europa, 10,52\% nos Estados Unidos e 5,26\% no México. Dos estudos brasileiros (42,10\%), a maioria $(21,06 \%)$ foi realizado na região sudeste, dos quais, 10,53\% foram feitos em São Paulo (Conhecimento e sentimentos das mulheres climatéricas sobre a doença coronariana; Qualidade de vida em mulheres no climatério atendidas na Atenção Primária) e 10,53\% em Minas Gerais (Educação em saúde para mulheres o climatério: impactos na qualidade de vida; Mulher no climatério: reflexões sobre o desejo sexual, beleza e feminilidade). E na região sul do país foram desenvolvidas 5,26\% das pesquisas (Incontinência urinária e disfunções sexuais em mulheres climatéricas de um grupo de promoção à saúde).

Quanto à formação acadêmica dos autores, nota-se que a maioria $(56,95 \%)$ foram publicados por enfermeiros, seguidos de 34,72\% publicações de médicos, fisioterapeutas (4,17\%), estatísticos (2,77\%) e biomédicos (1,39\%). Ao analisarse a abordagem, identificou-se que três $(15,8 \%)$ utilizaram a abordagem metodológica qualitativa e dezesseis $(84,2 \%)$ desenvolveram estudos com métodos quantitativos. Para melhor compreensão da temática, foram categorizados os achados para discussão.

\section{Categoria 1: Promoção da saúde para melhor Qualidade de Vida das mulheres climatéricas}

Frente às evidências encontradas, os estudos convergem para estratégias de promoção da saúde de mulheres que vivenciam o climatério. Desta forma, os autores descrevem sobre as necessidades e anseios vivenciados nesse período na vida de mulheres climatéricas. Isto pode fazer com que se sintam acolhidas e sejam melhores entendidas para que possam expor suas emoções, dúvidas e sensações a respeito desta fase, o que poderá proporcioná-las maiores informações e propiciar uma vida mais saudável contribuindo para a preservação de sua saúde.

O climatério caracteriza-se como uma fase de transformações sociais, fisiológicas e psicológicas. Portanto, constituise em um momento de grande vulnerabilidade para a mulher (Santos, 2016). No estudo de Silva, et al (2018), destaca-se a relevância de informações importantes sobre a saúde de uma parcela quase sempre negligenciada da população brasileira, as mulheres climatéricas.

Estas mulheres acabam atraindo a atenção da comunidade multidisciplinar como um grande problema de saúde. E atualmente, a incapacidade em vivenciar os sintomas da síndrome do climatério, especialmente a vasomotora, é a causa mais comum de encaminhamentos para centros de saúde (Yazdkhasti, Simbar, \& Abdi, (2015).

Ainda que a assistência à mulher deva buscar atender todas as suas necessidades de saúde, de forma integral e sem discriminações, na prática, essas ações nem sempre são desenvolvidas. É válido ressaltar também a escassez de estudos nesta área, o que remete a importância de enfocar melhor as questões relacionadas ao climatério, pois, torna-se necessário a identificação dos problemas vivenciados no período para que os mesmos sejam amenizados (Alves, Costa, Bezerra, Nakano, Cavalcanti, \& Dias, 2015).

O resultado do estudo de educação em saúde (ES) para mulheres no climatério revela a importância de avaliar se processos que promovam qualidade de vida (QV) em seus vários domínios. Devido ao caráter preventivo e promocional, a ES deve ser alvo constante de pesquisas para que tecnologias educacionais capazes de promover a saúde da mulher climatérica, sobretudo a QV, sejam desenvolvidas e aprimoradas (Freitas, Barbosa, Reis, Ramada, Moreira, Gomes, Vieira, \& Teixeira, 2016). 
Dados do estudo de Membrive, Granero-Molina, Salmerón, Fernández-Sola, López e Carreno, (2011), representam importantes fontes de hipóteses de trabalho para continuar investigando a relação entre a atividade profissional e a QV durante o climatério. Embora existam vários estudos acerca das condições clínicas associadas ao climatério, poucas pesquisas se dedicam à avaliação da autopercepção de saúde das mulheres durante esse período.

Ao avaliar a saúde da mulher climatérica e conhecer como ela própria percebe suas condições de saúde, Silva, et al (2018), conclui trabalhar na prevenção de morbidades permitindo uma melhor qualidade nessa fase da vida.

Por outro lado, considerando o papel influente do apoio social na melhoria da QV em mulheres na pós-menopausa, a participação de outros membros da família, especialmente cônjuges, em programas de promoção da saúde física e mental podem contribuir para uma melhoria da QV das mulheres (Yazdkhasti et al., 2015). Essa participação torna-se imprescindível para que essas mulheres se sintam apoiadas e compreendidas por familiares próximos nesta fase de difícil adaptação e compreensão.

\section{Categoria 2: A fase do climatério e o surgimento de doenças}

A síndrome do climatério é uma fase repleta de modificações e, consequentemente, essas modificações interferem na saúde biopsicossocial da mulher. É importante chamar a atenção para o fato de que alguns fatores de risco são mais prejudiciais para essas mulheres, como os níveis elevados de colesterol, diabetes e obesidade, fatores de riscos estes que se mostram mais prevalentes na população feminina como um todo (Mamede, Silva, \& Oliveira, 2019).

Alcântara, et al (2018) consideram que, embora o climatério seja um período importante e inevitável, deve ser encarado como um processo natural e fisiológico e não como doença. Muitas mulheres encontram-se despreparadas para vivenciá-lo, talvez pelo desconhecimento, pela qualidade da assistência oferecida à sua saúde e pela importância dada ao seu próprio eu. Dada a importância da temática e pela escassez de estudos que tratam das percepções, experiências e vivências das mulheres no climatério, vislumbra-se a necessidade de compreender este fenômeno.

Com essa nova fase acometendo o corpo da mulher, acaba tornando-as propensas às doenças que acompanham o período do climatério. De acordo com Raczkiewicz, et al (2017), mulheres na peri e pós-menopausa estão particularmente em risco de desenvolver a Síndrome Metabólica, pelo envelhecimento e perda do efeito protetor do estrogênio no corpo. No estudo do autor supracitado, o diagnóstico de hipertensão foi frequente, acometendo 57\% das mulheres participantes da pesquisa. Portanto, a hipertensão é um grave distúrbio metabólico que pode causar ataque cardíaco, derrame e insuficiência cardíaca.

Já a doença coronariana, foi considerada como a principal causa de morbimortalidade em vários países do mundo, especialmente em mulheres acima de 50 anos. Segundo Mamede et al. (2019), a doença coronariana impõe algumas modificações no cotidiano das mulheres, ainda que temporariamente, como o afastamento do trabalho, que, invariavelmente, provocam reações de estresse físico e emocional. O sentimento de impotência e inutilidade diante das restrições impostas pela doença, desde as tarefas mais elementares até o próprio autocuidado, representa uma ruptura na vida cotidiana dessas mulheres.

Dessa forma, faz-se necessário ter um olhar holístico sobre essas mulheres visto que doenças já existentes possam interferir ainda mais na sintomatologia da fase do climatério, influenciando nos aspectos sociais, culturais e biológicos. Podendo trazer consigo diversas implicações para a vida, ocasionando mais dificuldades de compartilhamento dos seus sentimentos, fazendo com que se sintam desamparadas, desprotegidas e isoladas.

\section{Categoria 3: O impacto do climatério na sexualidade e feminilização}

Os estudos apontaram que as mulheres climatéricas sofrem grandes alterações fisiológicas que variam de mulher para mulher na sintomatologia e intensidade, podendo gerar consequências diretas que podem afetar o bem-estar biopsicossocial. 
Dentre as áreas afetadas pelo climatério, a sexualidade foi evidenciada como um fator que necessita de atenção pelos profissionais de saúde, pois é reconhecida como um dos pilares da QV.

Mulheres climatéricas são constantemente martirizadas diante do mito da perda do seu desejo sexual associado ao envelhecimento. Isso pode fazer com que algumas mulheres reproduzam todos os preconceitos, mitos e medos que circulam na sociedade sobre o climatério. Com isso, essas mulheres podem se sentir menos atraentes e desejáveis, podendo, assim, prejudicar seu convívio familiar, social, conjugal e sexual (Valença, Filho, Germano, 2010).

Santos, Siqueira e Nascimento (2015) abordam que a síndrome do climatério pode apresentar sinais e sintomas transitórios, permanentes e de prevalência altamente variável, uma vez que, é diretamente influenciada por fatores ligados ao meio e a singularidade de cada mulher. Entre os diferentes sintomas que a mulher pode enfrentar, alguns podem influenciar a sua vida sexual. É necessário estender o olhar frente a presença de problemas sociais, como a síndrome do ninho vazio, doenças instaladas, incompreensão no relacionamento conjugal e entre outros, que podem desencadear grandes repercussões na QV e autoestima.

Percebe-se, então, que a questão do desejo sexual no climatério não segue uma linearidade fácil de ser mensurada. Sendo assim, um estudo com o objetivo de verificar a associação entre a intensidade dos sintomas no climatério e o padrão de desempenho sexual de mulheres, apontaram que o padrão de desempenho sexual elevado pode estar associado a uma menor intensidade dos sintomas. Foi concluído através desse estudo que a prática da atividade física pode estar associada com uma menor intensidade dos sintomas do climatério e uma elevação nos padrões de desempenho sexuais (Alves, et al., 2015).

O hipoestrogenismo, condição fisiológica do climatério, influência a lubrificação vaginal tornando o período de excitação mais demorado e menos intenso, sendo determinante no aparecimento da dispareunia, trazendo como consequência, modificação da resposta orgástica feminina (Alves et al., 2015). Tais fatores trazem consequências para vida sexual das mulheres, podendo afetar consequentemente a feminilidade.

Nesse sentido, a equipe multidisciplinar pode orientar a mulher que enfrenta esses problemas sobre a adoção de hábitos de vida saudáveis como a prática de atividades físicas e alimentação saudável, podendo trazer melhoras consideráveis para sua qualidade de vida. Além disso, doenças associadas podem influenciar também nos sintomas pertinentes ao climatério e diminuição do desempenho sexual, tornando necessário o acompanhamento dessa mulher como um todo.

No estudo qualitativo de satisfação sexual entre mulheres de meia-idade de Thomas, et al. (2019), descobriram que: sentir-se atraente foi um importante motivo para a atividade sexual; foram comuns alterações na aparência, especialmente ganho de peso e alterações nas mamas; e como as mulheres reagiram a essas mudanças impactaram sua atividade sexual e satisfação. Ainda nesse estudo, foram observados que mulheres que se sentiam inseguras e autoconscientes sobre seus corpos notaram que essas preocupações tiveram um impacto negativo em sua satisfação sexual. Enquanto as mulheres que se sentiam confiantes e atraentes discutiam melhor satisfação sexual, mesmo diante das mudanças corporais. Desse modo, observa-se uma importante relação entre a autoestima, confiança e aceitação da mulher com satisfação e qualidade da sua sexualidade.

De acordo com Afsahri et al. (2018), apontam uma relação significativa entre a sexualidade e a qualidade do sono. Ao considerar os tipos de qualidade do sono, excitação sexual, lubrificação, orgasmos, satisfação, dor durante a relação e o desejo sexual, observou-se uma associação significativa da qualidade do sono e da insônia.

Para Valença, et al (2010), o tipo de companheiro é capaz de revelar muitos aspectos da batalha travada entre a mulher e sua sexualidade. Relações de poder, de atividade-passividade, manifestações e papéis culturais são apenas alguns fatores a serem considerados na tríade do matrimônio, sexualidade e climatério. Pressupõe-se que algumas mulheres vivenciarão o climatério com maior dificuldade se forem estabelecidas poucas ou nenhuma intimidade na relação conjugal ou parceira. Desse modo, a intimidade com o parceiro que a mulher se relaciona é de extrema importância para que a mesma se sinta bem com as mudanças enfrentadas durante esta fase. 
Assim, a informação em saúde, sobre o seu corpo, autocuidado e relacionamento com o parceiro tornam-se aspectos fundamentais para essas mulheres a fim de vencer os conflitos que permeiam essa fase feminina. A mulher no período do climatério enfrenta diversos fatores que podem alterar sua atividade e apetite sexual, não somente a fase climatérica em si, mas todo o contexto em que essa mulher vive.

Categoria 4: O climatério e as repercussões no trabalho

O climatério pode não causar problemas significativos para algumas mulheres, mas para outras é conhecido por apresentar dificuldades consideráveis em suas vidas pessoais e de trabalho (Jafari et al., 2017). Observa-se através de contexto histórico que as mulheres começaram a ganhar espaço no ambiente de trabalho há pouco tempo. Apesar do trabalho ter trago melhor qualidade de vida para as mulheres, é perceptível que há uma dificuldade no reconhecimento e nas condições de trabalho para elas, mesmo as mulheres formando grande parte da força de trabalho atualmente.

Segundo Cardoso \& Camargo (2015) a atividade de trabalho tem um papel importante na participação social, além de contribuir para a autoestima e para a independência do indivíduo. De acordo com Oliveira, Jesus e Merighi (2008) ter uma profissão é indicativo de valorização para as mulheres. A possibilidade da mulher de estar cercada de pessoas diferentes do seu ciclo familiar e com diversas experiências de vida, torna mais favorável quando se tem um trabalho fora de casa. Madalozzo, Martins e Shiatori (2010) consideram que ter dois ambientes de trabalho podem prejudicar a produtividade da mulher ao exercer todas as funções delegadas, favorecendo o surgimento de vulnerabilidade de saúde e bem-estar.

É de conhecimento do senso comum a discriminação que as mulheres sofrem no seu cotidiano e, principalmente, no mercado de trabalho, sendo consideradas frágeis e inferiores (Costa, 2014). O preconceito e a discriminação produzem efeitos danosos na saúde da mulher de modo geral, ainda assim a discriminação racial e de gênero têm repercussões nos serviços de saúde, inclusive no que diz respeito à assistência de enfermagem (Silva \& Falleiros et al., 2015).

Segundo Abedzadeh, Taebi, Sadat, e Saberi (2011) as mulheres nas sociedades desenvolvidas tinham uma maior qualidade de vida em comparação com aquelas em sociedades em desenvolvimento, além disso, também foi observado maior QV em comparação com as donas de casa. Embora seja relatado que mulheres trabalhadoras tenham melhor QV, trabalhar não poderia ser um preditor da mesma, visto que, outros fatores podem interferir no seu ambiente de trabalho gerando menor QV.

Quase metade das mulheres participantes no estudo de Gujski, et al (2017), experimentaram um alto nível de estresse no local de trabalho. A maioria das participantes apresentaram estresse no ambiente de trabalho causado por contatos sociais, evidenciando a importância de ter uma boa relação com os colegas de trabalho para que haja menos estresse nesse local e consequentemente, uma diminuição da intensidade dos sintomas apresentados na fase do climatério.

É necessário que os gestores tenham ciência de que algumas mulheres que vivenciam o período do climatério sentem prejuízo na relação de confiança e podem apresentar um comportamento incomum, tendo a necessidade de pequenas pausas para maior conforto frente aos sintomas (Jafari, et al., 2017). Nesse sentido, proporcionar melhorias no ambiente de trabalho para as funcionárias pode trazer benefícios para a mulher, para o trabalho e seu rendimento diante de suas responsabilidades.

Em contrapartida, o trabalho coletivo viabiliza a troca de experiências entre as mulheres e permite que elas percebam que não estão sozinhas, essa percepção aliada ao uso de dinâmicas de grupo e espaços de confraternização pode facilitar a integração e contribuir para a melhoria das relações sociais (Freitas et al., 2016). Desse modo, é visível que um ambiente de trabalho acolhedor e uma boa convivência com o meio torna-se necessário para que a mulher se sinta confiante, produtiva e menos solitária.

Nesse contexto, é importante que sejam elaborados meios de educação em saúde nas empresas a fim de orientar, intervir e contribuir para a promoção e manutenção das relações de trabalho, igualdade de gênero e desconfortos vivenciados pelas mulheres climatéricas. Tais estratégias podem contribuir para os problemas apontados e minimizar os sintomas comuns deste período, além de proporcionar espaços para discussões sobre diversos assuntos que permeiam essa fase e a vida das 
mulheres.

\section{Conclusão}

A avaliação da saúde da mulher climatérica e o conhecimento em relação a sua saúde tem sido apontada como indicadores de qualidade de vida para fins de promoção da saúde. A qualidade do sono, a alimentação equilibrada, uma vida sexual satisfatória, relações familiares e conjugais acolhedoras, assim como a prática de atividade física e melhorias no ambiente de trabalho foram evidenciadas nos estudos selecionados nesta revisão e que contribuem para a melhoria da qualidade de vida das mulheres.

Destaca-se a relevância no investimento em pesquisa na temática e a integralidade da assistência às mulheres. Fatores de risco prejudiciais à saúde foram apontados no âmbito físico, emocional e social. Foram relatados dentre os fatores físicos, hipertensão arterial sistêmica, doença coronariana, elevação de colesterol, diabetes e obesidade. No aspecto emocional destacase o estresse por se sentirem desamparadas, desprotegidas e isoladas baseados nos significados elaborados a partir das questões sociais e culturais. Na esfera social, a desigualdade entre homens e mulheres no trabalho foi evidente, além da falta de compreensão e acolhimento no ambiente laboral, repercutindo no enfrentamento dos sintomas da fase.

O estudo contribui com evidências atuais possibilitando criação de estratégias aos profissionais na assistência às demandas dessas mulheres. É necessário maior esclarecimento para que as mulheres tenham uma compreensão sobre a fase fisiológica, emocional e social do climatério. Nesse sentido, destaca-se a necessidade de futuros trabalhos na área que amplie possibilidades para maior divulgação do tema, bem como pesquisas que avaliem a percepção dos profissionais na rotina dos serviços e na assistência às mulheres nessa fase.

\section{Referências}

Abedzadeh, M., Taebi, M., Sadat, Z., \& Saberi, F., (2011). Assessment of Quality of Life in Menopausal Periods: A Population Study in Kashan. Iranian Red Crescent Medical Journal, 13(11).

Afsahri, P., Salehnejad, Z. \& Hekma, K. (2018). Do Sleeping Disorders Impair Sexual Function in Married Iranian Women of Reproductie Age? Results from a Cross-Sectional Study. Psychiatry Journal, 1045738, 7.

Alcântara, D. S. (2018). A vivência do Climatério por Mulheres Atendidas em uma Unidade Básica de Saúde no Município de Gurupi-TO. Amazônia: Science \& Health, 6(1), 43-47.

Alves, E. R. P., Costa, A. M., Bezerra, S. M. M. S., Nakano, A. M. S., Cavalcanti, A. M. T. S., \& Dias, M. D., (2015). Climatério: A Intensidade dos Sintomas e o Desempenho Sexual. Texto Contexto Enfermagem, 24(1).

Bariola, E., Jack, G., Pitts, M., Riach, K. \& Sarrel, P. (2017). Employment Conditions and Work-Related Stressors are Associated with Menopausal Symptom Reporting Among Perimenopausal and Postmenopausal Women. Menopause, 24, 247-251.

Ercole, F. F., Melo, L. S., \& Alcoforado, C. L. G. C., (2014). Revisão Integrativa versus Revisão Sistemática. REME-Revista Mineira de Enfermagem, 18(1).

Freitas, E. R. (2016). Educação em Saúde para Mulheres no Climatério: Impactos na Qualidade de Vida. Reprodução e Climatério, $31(1)$, 37-43.

Instituto Brasileiro de Geografia e Estatística - IBGE, (2015). Pesquisa Nacional por Amostra de Domicílios - PNAD/IBGE. O Instituto.

Gujski, M. (2017). Stress at the Place of Work and Cognitive Functions Among Women Performing Intellectual Work During Peri- and post- Menopausal Period. International Journal of Occupational Medicine and Environmental Health, 30(6), 943-961.

Hunter, M. S., Hardy, C., Norton S. \& Griffiths A. (2016). Study Protocol of a Multicentre Randomised Controlled Trial of Selfhelp Cognitive Behaviour Therapy for Work ith Menopausal Symptoms. Maturitas, 92, 186-192.

Jafari, M. (2017). Risk Assessment: Factors Contributing to Discomfort for Menopausal Women in Workplace. JMM - Journal of Menopausal Medicine, 23(2), 85-90.

Madalozzo, R., Martins, S. R., \& Shiatori, L., (2010). Participação no Mercado de Trabalho Doméstico: Homens e Mulheres têm Condições Iguais? Revista Estudos Feministas, 18(02): 352.

Mamede, M. V., Silva, L. D. C. \& Oliveira, B. S. (2019). Conhecimento e Sentimentos das Mulheres Climatéricas sobre a Doença Coronariana. REME Revista Mineira de Enfermagem, 23, 1167. 
Research, Society and Development, v. 10, n. 8, e47310817514, 2021

(CC BY 4.0) | ISSN 2525-3409 | DOI: http://dx.doi.org/10.33448/rsd-v10i8.17514

Martínez-Garduno, M. D. (2016). Intervención Educativa de Enfermería para Formentar el Autocuidado de la Mujer Durante el Climaterio. Enfermería Universitaria, 13(3), 142-150.

Matsuzaki, K., Uemura, H., \& Yasui, T., (2014). Associations of Menopausal Symptoms with Job-Related Stress Factors in Nurses in Japan. Maturitas, 79(1).

Membrive, J. M., Granero-Molina, J., Salmerón, M. J. S., Fernández- Sola, C., López, C. M. R., \& Carreno, T. P., (2011). Qualidade de Vida em Mulheres Climatéricas que trabalham no Sistema Sanitário e Educativo. Revista Latino-Am Enfermagem, 19(6).

Oliveira, A. R. (2019). Promoção à Saúde da Mulher: Desmistificando o Climatério. Brazilian Journal of Development, 5(10), 21431-21442.

PNAD Contínua: Número de idoso cresce 18\% em 5 anos e ultrapassa 30 milhões em 2017. Laboratório de Demografia e Estudos Populacionais, 2018.

Raczkiewicz, D. (2018). Metabolic Syndrome in Peri- and Post Menospausal Women Performing Intellectual Work. Annals of Agricultural and Environmental Medicine, 25(4), 610-615.

Santos, A. A. S., (2016). Percepção das Mulheres no Município de Paulo Afonso, na Bahia, Sobre as Mudanças Corporais e Emocionais no Período do Climatério. UNIFAP, 6(1).

Santos, A. C. B. C., Siqueira, J. A. S., \& Nascimento, I. N. A. (2015). Alterações na Capacidade Funcional em Mulheres Menopausadas. Interscientia, 3(2).

Schmalfuss, J. M., Sehnem, G. D. \& Rossetto, M. (2020). A Sexualidade de Mulheres Durante o Climatério: Percepções e Vivências. Research, Society and Development, $9(9)$.

Silva, G. F. \& Moura, M. A. V. (2015). Influências do Climatério para o Envelhecimento na Percepção de Mulheres Idosas: Subsídios para a Enfermagem. Revista Eletrônica de Enfermagem, 17(3).

Silva, V. H. (2018). Fatores Associados à Autopercepção Negativa de Saúde em Mulheres Climatéricas. Revista Ciência \& Saúde Coletiva, 23(5), 1611-1620.

Soares, G. R. S., Cortez, E. A., Silva, R. M. C. R. A., Sá, S. P. C., \& Simoes, S. M. F., (2015). O Cuidar em Saúde Mental: Contribuições Fenomenológicas Acerca de Mulheres Trabalhadoras em Situação de Climatério. Revista Cubana de Enfermería, 31(2).

Thomas, H. N. (2019). Body Image, Attractiveness, and Sexual Satisfaction Among Midlife Women: A Qualitative Study. Journal of Woman's Health, 28(1), 100-106.

Valença, C. N., Filho, J. M. N., Germano, R. M., (2010). Mulher no Climatério: Reflexões sobre Desejo Sexual, Beleza e Feminilidade. Saúde e Sociedade, 19(2).

Yazdkhasti, M., Simbar, M., \& Abdi, F., (2015). Empowerment and Coping Strategiesin Menopause Women: A Review. Iran Red Crescent Med Jornaul, 17(3). 